\title{
Period doubling and chaos in large area Josephson junctions induced by rf signals
}

\section{Olsen, O. H.; Samuelsen, Mogens Rugholm}

\section{Published in:}

Applied Physics Letters

Link to article, DOI:

10.1063/1.95971

Publication date:

1985

Document Version

Publisher's PDF, also known as Version of record

Link back to DTU Orbit

Citation (APA):

Olsen, O. H., \& Samuelsen, M. R. (1985). Period doubling and chaos in large area Josephson junctions induced by rf signals. Applied Physics Letters, 47(9), 1007-1009. https://doi.org/10.1063/1.95971

\section{General rights}

Copyright and moral rights for the publications made accessible in the public portal are retained by the authors and/or other copyright owners and it is a condition of accessing publications that users recognise and abide by the legal requirements associated with these rights.

- Users may download and print one copy of any publication from the public portal for the purpose of private study or research.

- You may not further distribute the material or use it for any profit-making activity or commercial gain

- You may freely distribute the URL identifying the publication in the public portal 


\title{
Period doubling and chaos in large area Josephson junctions induced by rignals
}

\author{
O. H. Olsen \\ Systems Engineering, Niro Atomizer a/s, Gladsaxevej 305, DK-2860, Denmark \\ M. R. Samuelsen \\ Physics Laboratory I, The Technical University of Denmark, DK-2800, Denmark
}

(Received 17 July 1985; accepted for publication 14 August 1985)

\begin{abstract}
The influence of an applied rf signal on the emitted radiation from a large area Josephson junction is examined. A model of the system is presented in the framework of the one-dimensional sineGordon equation. The model linearizes for small and large values of the amplitude of the applied signal. In the intermediate regime lower and upper threshold values (for the amplitude) for transition to chaos are found. Feigenbaum period doubling (period-doubling bifurcation cascade) appears when chaos is approached for increasing amplitude. The findings are supported experimentally.
\end{abstract}

Chaotic phenomena in deterministic systems have recently attracted a great deal of interest. Especially the chaotic behavior of physical systems in which the spatial degrees of freedom cannot be neglected is being examined. Among many examples are the free-surface modes of a circular fluid layer forced vertically, ${ }^{1}$ optical turbulence, ${ }^{2}$ and sine-Gordon systems. ${ }^{3-6}$ The latter examples cover condensed matter systems (such as Josephson junctions), charge density wave materials, etc. The investigations in Ref. 3 concern the influence of a spatially inhomogeneous, oscillating driving force on a sine-Gordon system with outflow boundary conditions while Refs. $4-6$ concern a spatially homogeneous, oscillating driving force on a similar system with periodic boundary conditions. Both of these systems have periodic as well as chaotic responses. However, these systems are very difficult to realize experimentally in Josephson physics.

In the present letter we focus on large area Josephson junctions (overlap tunnel junctions) irradiated with $\mathrm{rf}$ signals (without applied dc current). This has been realized experimentally. ${ }^{7}$ The microwave pump signal is applied to one end of the junction. This system is modeled by a perturbed onedimensional sine-Gordon equation with appropriate boundary conditions describing the influence of the $\mathrm{rf}$ signal. The model equation can be solved approximately for small and large values of the amplitude of the applied rf signals. In the intermediate regime the system has been examined numerically and chaotic regimes have been found as well as Feigenbaum period doubling. These findings are supported experimentally.

The dynamics of a long Josephson tunnel junction is assumed to be governed by a perturbed sine-Gordon equation $^{8}$ :

$$
u_{x x}-u_{t t}=\sin u+\alpha u_{t},
$$

where $u$ is the phase difference between the two superconducting films. The spatial variable is measured in units of the Josephson penetration depth $L_{J}=(\hbar / 2 e d \mu J)^{0.5}$ and the time in units of the reciprocal plasma frequency $\Omega_{0}^{-1}$, where $\Omega_{0}=(2 e J / \hbar C)^{0.5}$. Here $J$ is the Josephson current density, $d$ is the magnetic thickness of the barrier, and $C$ is the capacitance per unit area. The loss parameter $\alpha$ is defined through the relation $\alpha=G(\hbar / 2 e J C)^{0.5}$, where $G$ is the shunt conductance per unit area.

With an oscillating magnetic field $H_{e} \sin \Omega t$ applied to one end of the junction, perpendicular to the length of the junction and parallel to the plane of the barrier, the boundary conditions for the phase difference $u$ at the ends are

$$
\begin{aligned}
& u_{x}(l)=a \sin \Omega t, \\
& u_{x}(0)=0,
\end{aligned}
$$

where $a=H_{e} / J L_{J}$ and $l=L / L_{J}$ are the normalized magnetic field strength and junction length, respectively.

An approximate solution to Eqs. (1) and (2) can easily be found for $a<1$ and $a>1$ corresponding to $\left|u_{t}\right|<1$ and $\left|u_{t}\right|>1$, respectively. In these limits we approximate $\sin u$ with $n u$, where $n=1$ for $a<1$ and $n=0$ for $a>1$. The following Ansatz is applied: $u(x, t)=u_{+} e^{i k x-i \Omega t}+u_{-} e^{-i k x-i \Omega t}$ (i.e., the solution consists of traveling wave trains). Inserting the Ansatz in Eq. (1) gives the dispersion relation $k^{2}=\Omega^{2}-n+i \alpha \Omega$, while the boundary conditions Eq. (2) give:

$$
\begin{aligned}
& u_{+}=u_{-}, \\
& u_{+}=a /(2 k l \sin k l),
\end{aligned}
$$

so that the final approximate solution is given by

$$
u=a e^{-i n t} \cos k x /(-k \sin k l) \text {. }
$$

Note that $k$ is complex. Thus the solution presented in a phase plane is an ellipse.

In the following we present results concerning the intermediate regime. Equations (1) and (2) have been solved numerically. In the numerical solution we have applied an approximation based on a stabilized leap frog scheme. Throughout this letter we have chosen flat initial conditions, i.e., $u(x, 0)=0$ and $u_{t}(x, 0)=0$. Further, the frequency $\Omega=0.8$, the loss parameter $\alpha=0.2$, and the normalized length of the junction $l=5$ are held fixed. Each solution has typically been followed to $t=4000$; the transients die out after $t=1000$. The primary diagnostic tools are phase plane trajectory plots [e.g., $u_{t}(l)$ vs $\left.u(l)\right]$ and Poincare' maps. The latter has been obtained by plotting $u_{t}(l)$ vs $u(l)$ at equally spaced time intervals corresponding to the positive zero 


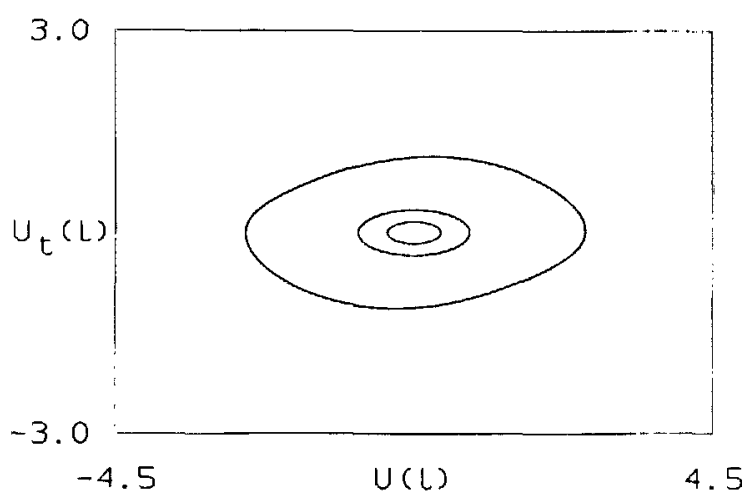

(a)

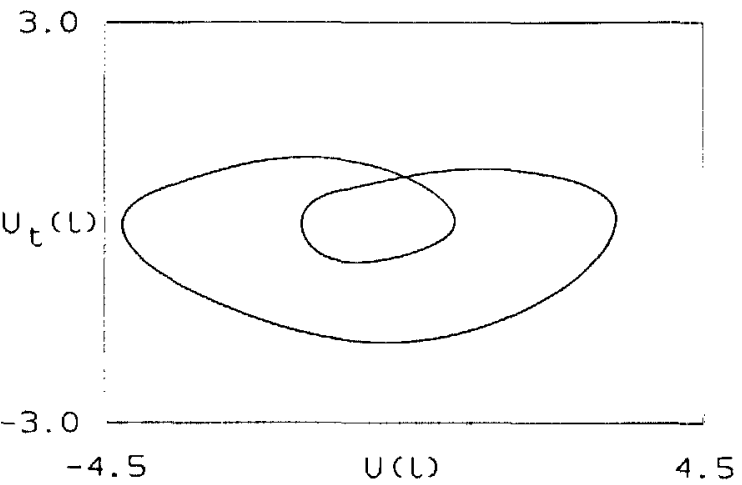

(b)

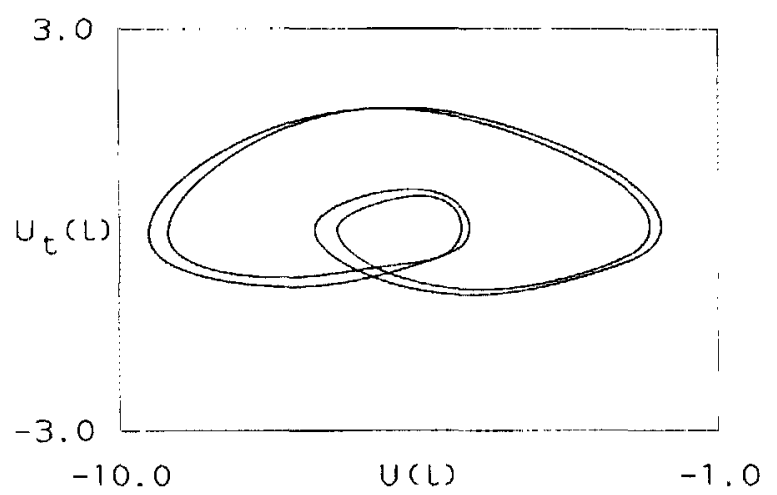

(c)

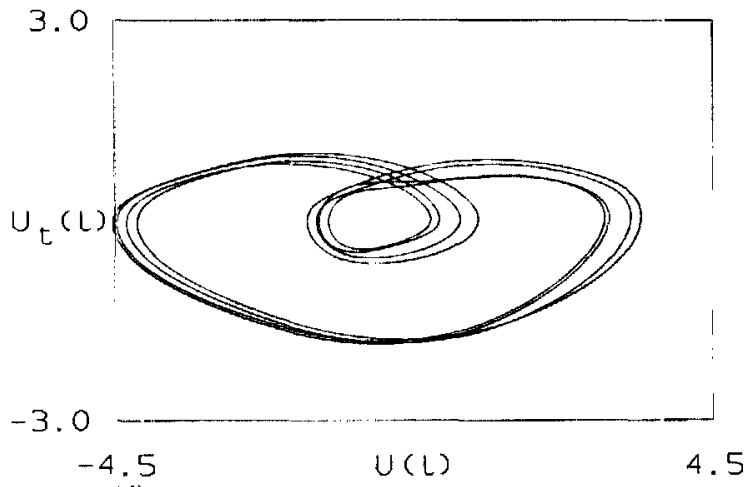

(d)

FIG. 1. Phase plane plots $u_{\mathrm{r}}(l)$ vs $u(l)$ for values of the amplitude below the lower threshold value for the chaotic regime. Parameter values $\Omega=0.8$ and $l=5$. In (a) the solution is single periodic ( $a=0.25,0.5$ and 1.25 from center and out), in (b) double periodic $(a=1.525)$, in (c) quadruple periodic $(a=1.54)$, and in (d) octuble periodic $(a=1.5475)$.

crossings of the applied rf signal. Furthermore, we have folded the $u$ values onto a $2 \pi$ interval. Remark that $u_{t}$ corresponds to the measured voltage. In Fig. 1 we show the solution to Eqs. (1) and (2) for increasing amplitude $a$. The results are presented in a phase plane plot for $t$ in the interval from 3000 to 4000 corresponding to more than 100 periods of the applied rf signal. In Fig. 1(a) the inner ellipsis corresponds to $a=0.25$ and $a=0.5$, respectively. This is in agreement with the analysis above. The third curve corresponds to $a=1.25$; the symmetry is broken due to the nonlinearity. In Figs. 1 (b)-1(d) are shown examples of stable solutions in a perioddoubling sequence. This is the beginning of an infinite sequence of bifurcations leading to chaos. We have detected two more bifurcstos: corresponding to periods $\mathbf{1 6}$ and 32 . Furthermore, we have roughly estimated Feigenbaum's universality constant to 4.5 which is close to the real number (4.6692). In Fig. 2 we show Poincare' maps for the amplitude just below the threshold to chaos $(a)$ and in the chrotic regime (b). The threshold value is ound to be close to $a=1.555$. In Fig. 2(a) a strange attractor is formed, while in Fig. 2(b) the response is nonperiodic, i.e., chaotic. In contrast to the solution in Fig. 2(a) the phase $u$ jumps between $2 \pi$ bands in the solution in Fig. 2(b). Finally, in Fig. 3 we show the transition from the chaotic regime to the regime periodic solutions. We have not found a Feigenbaum bifurcation sequence in this case. The transition to the regime of periodic solutions occurs continuously. In this case the threshold value is hard to determine because of the difficulty in distin-
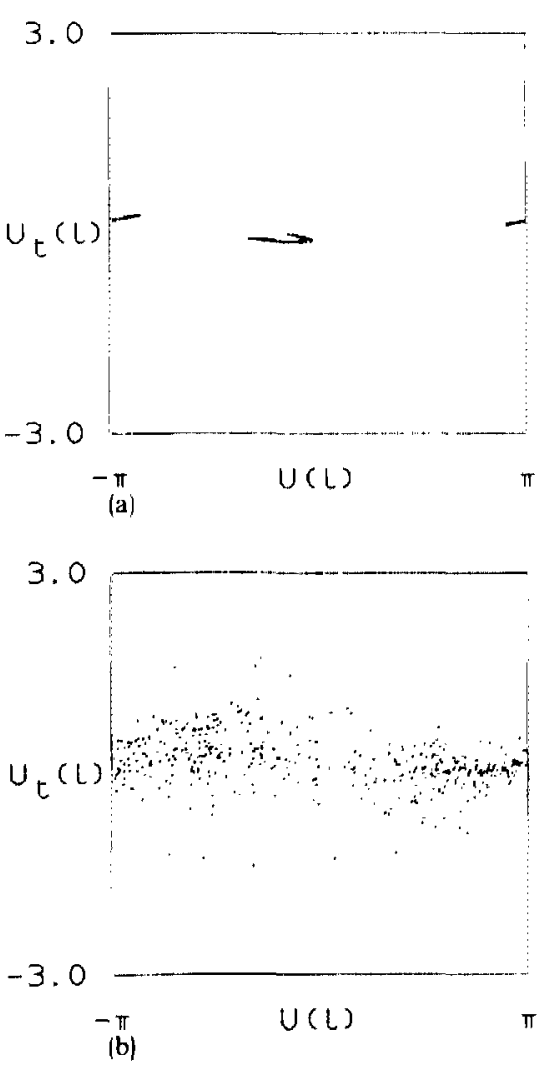

FIG. 2. Poincare' maps for values of the if amplitude just below the chaotic regime (a) and in the chaotic regime (b). In (a) $(a=1.55)$ a strange attractor is formed while in (b) $(a=1.6)$ nonperiodic response (chaos) is found. 


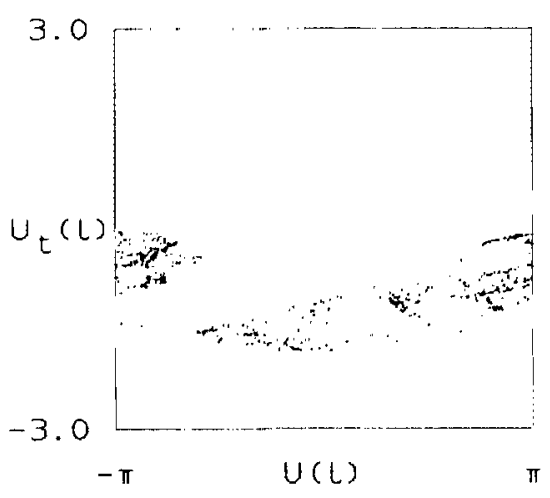

(a)

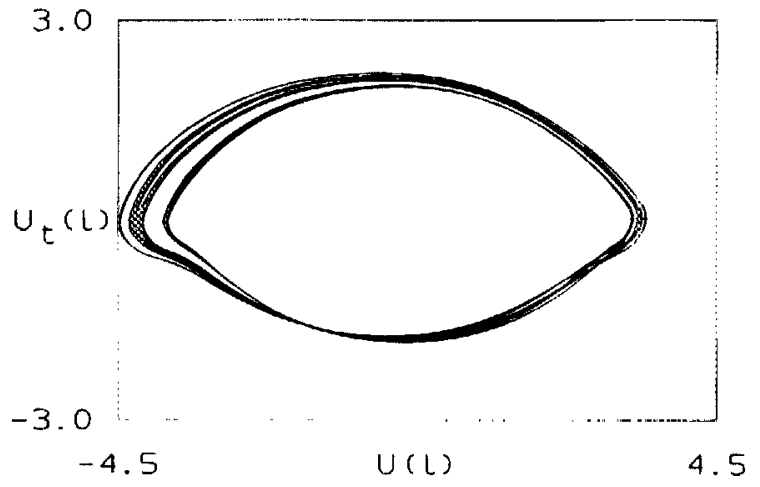

(b)

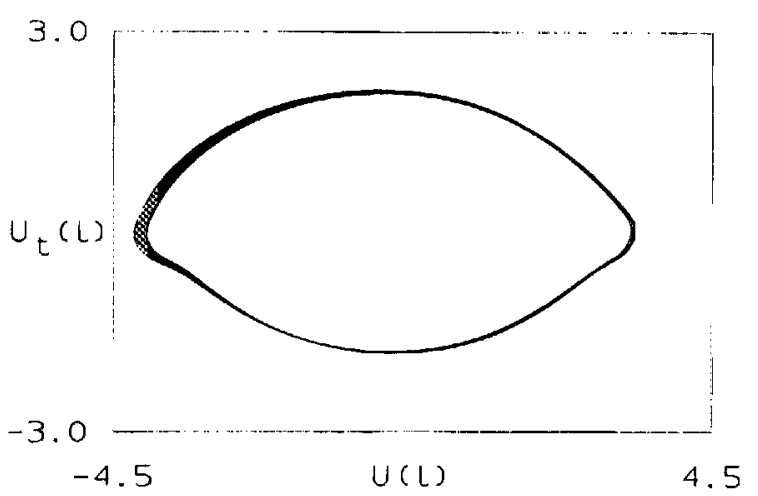

(c)

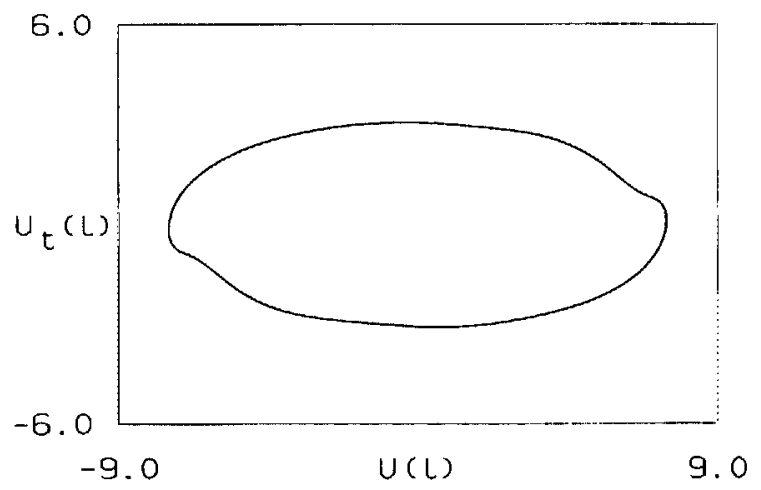

(d)

FIG. 3. Poincare' map and phase plane plots illustrating transition from the chaotic regime to the regime of periodic solutions. In (a) $(a=5)$ the response is chaotic, in (b) and (c) $(a=5.525$ and 5.5275) either strange attractors or chaos is formed, while in (d) $(a=6)$ a periodic solution found.

guishing between chaotic response and response described by strange attractors (i.e., stable long periodic motions). However, from an experimental point of view there will be no difference. Our estimate of the threshold values is $a=5.5$. In Fig. 3(a) the response is chaotic while Figs. 3(b) and 3 (c) show the transition to single periodic solutions. For larger values of $a$, say $a=10$., the phase plane plot becomes ellipse-like in accordance with the analysis above. We note that in Ref. 7 the first period doubling has been observed experimentally for a junction of the same normalized length but with a slightly smaller loss factor. The following period doublings will probably be very difficult to detect.

In conclusion, we note that the aim of this letter is to present a simple system which possesses periodic as well as chaotic responses and is possible to realize experimentally. Preliminary results show good agreement between the model and experiment. So this system is inviting for experimentalists devoting effort to investigation of chaotic noise. At present we are investigating the influence of the chaos on the spatial modes in the junction- the chaos only results in a small loss of mode locking, ${ }^{10}$ i.e., the structures in the nonchaotic regime are preserved. Furthermore, we examine the system for hysteresis, which we do not expect to find.

'S. Ciliberto and P. J. Gollup, Phys. Rev. Lett. 52, 922 (1982).

${ }^{2}$ K. Ikeda, H. Daido, and O. Akimoto, Phys. Rev. Lett. 45, 709 (1980).

${ }^{3}$ J. C. Eilbeck, P. S. Lomdahl, and A. C. Newell, Phys. Lett. A 87, 1 (1981).

${ }^{4}$ A. R. Bishop, K. Fesser, P. S. Lomdahl, W. C. Kerr, M. B. Williams, and S. E. Trullinger, Phys. Rev. Lett. 50, 1095 (1983).

${ }^{5}$ A. R. Bishop, K. Fesser, P. S. Lomdahl, and S. E. Trullinger, Physica D (Utrecht) 7, 259 (1983).

'D. Bennett, A. R. Bishop, and S. E. Trullinger, Z. Phys. B 47, 265 (1982)

${ }^{7} Y$ u. Ya. Divin, J. B. Hansen, J. Mygind, O. H. Olsen, N. F. Pedersen, and M. R. Samuelsen, "Chaos and subharmonic generation in long Josephson junctions" (unpublished).

${ }^{8}$ D. W. MacLaughlin and A. C. Scott, Phys. Rev. A 18, 1652 (1978).

${ }^{9}$ R. K. Dodd, J. C. Eilbeck, J. D. Gibbon, and H. C. Morris, Solitons and Nonlinear Wave Equations (Academic, London, 1982), p. 581.

${ }^{10} \mathrm{~A}$. R. Bishop, J. C. Eilbeck, I. Satija, and G. Wysin, "Pattern selection and low-dimensional chaos in dissipative many degree-of-freedom systems" (unpublished). 